Research Article

\title{
The Influences of Squeezed Inviscid Flow between Parallel Plates
}

\author{
Sobia Akbar (D) and Azad Hussain \\ Department of Mathematics, University of Gujrat, Gujrat 50700, Pakistan \\ Correspondence should be addressed to Sobia Akbar; 18026109-004@uog.edu.pk \\ Received 11 November 2020; Revised 18 February 2021; Accepted 30 March 2021; Published 22 April 2021 \\ Academic Editor: Francesco Aggogeri \\ Copyright (C) 2021 Sobia Akbar and Azad Hussain. This is an open access article distributed under the Creative Commons \\ Attribution License, which permits unrestricted use, distribution, and reproduction in any medium, provided the original work is \\ properly cited. \\ Purpose. The main purpose of this study is to investigate the unsteady flow behavior of second-grade inviscid fluid between parallel
plates. The effects on the flow are explored through modeling of continuity, momentum, and energy equations. Graphical and
tabular exploration has been made to analyze the impact of several influential variables on the dimensionless temperature and
velocity profiles. Three-dimensional graphs and stream lines are also mentioned. Design/Approach/Methodology. The governing
equations have been metamorphosed into nonlinear ordinary differential equations by using suitable transformation which is the
main focus of the study. To approach the solution of the problem numerically, we have used the numerical method such as
shooting technique along with Runge-Kutta method is implemented. Findings. The graphs for the squeezing number, Prandtl
number, and Eckert number are decreasing by increasing the values of these parameters. The graphs of skin friction coefficient and
Nusselt number are increasing by changing the values of both parameters. Originality/Value. The significances of an unsteady
squeezed flow of a nonviscous second-grade fluid between parallel plates by using boundary layer phenomenon are discussed.
}

\section{Introduction}

Some important applications of non-Newtonian fluids are introduced to enhance the research interest in food preservation, polymeric substitutions, nuclear fuels, liquid metals, paints, and blood flow. In non-Newtonian fluids, mixed convection phenomenon has enchanted scientific experts because of its momentousness real-world applications, for example, solar energy, electronic appliances cooled with fans, and cooling of nuclear reactors. Complicated comparison of viscous fluids is formed due to strange nature between shear stress and strain rate in such fluids. The criterion of viscoelasticity contributed further complexities in the governing equations at the time of comparison with Navier-Stokes equations. Most of the investigations [1-25], worked on such problems by assuming different types of flows and effects on various fluids. The flow squeezed between parallel walls happens in many biological and industrial systems. The nonsteady viscous flow fluid squeezed between parallel plates is a great subject of interest in hydrodynamic machines due to their motion normal to their own surfaces. The initiate work and the fundamental formulation of under lubrication squeezing flows were assumed by Stefan [14]. In previous literature, over few decades, the flow squeezed by elliptic plates was discussed by Reynolds [15] while Archibald [16] suggested the same inquisition for rectangular plates.

The evaluation of boundary layer squeezed flow is an interesting research matter due to its wide range of applications in industry and engineering. The most common scientific and engineering applications are in the drawing of plastic wires and films, extrusion of a polymer in a meltspinning process, manufacturing of foods, crystal growing, liquid film in condensation process, electrochemical process, paper and glass fiber production, thermal energy storage, electronic chips, flow through filtering devices, food processing, cooling towers, marine engineering, hydro towers, distillation columns, and so on. The viscosity and thermic conductivity are presumed as a function of temperature. Unsteadiness is the loss of equilibrium with environment, usually with an affection of almost falling, or the consequences of bumping into objects. There are numerous reasons for unsteadiness, together with the problems in the cerebral or cerebellar sections of the spinal cord, brain, inner 
ear, or vestibular system. Unsteady flow of the fluid is the one where properties of the fluid vary with time. It is worthless to say that any beginning procedure is unsteady.

Now, the development in industries has motivated the researchers to discover non-Newtonian fluids properties in a more organized way. In nature, a number of fluids show non-Newtonian behavior, i.e., slurries, honey, glue, gels, toothpaste, ketchup, etc. Various paradigms of unsteadiness can be found from our daily life like the flow of water out from a tap which has been just opened. This is unsteady flow in the start, but it becomes steady with time. In the current problem, the boundary layer approximation is utilized to construct an unsteady second-grade fluid flow model. The obtained coupled partial differential equations are simplified by using suitable mathematical techniques. The dimensionless equations are being solved by using numerical techniques, i.e., shooting technique. A comprehensive graphical and tabular study is constructed to check the convergence of the obtained results.

\section{Mathematical Description of the Flow Phenomenon}

The stress tensor [14] for the current problem is given by

$$
T=-\mathrm{PI}+\mu A_{1}+\alpha_{1} A_{2}+\alpha_{2} A_{1}^{2} .
$$

We have restriction as

$$
\mu \geq 0, \alpha_{1} \geq 0, \alpha_{1}+\alpha_{2}=0, \quad \alpha_{1}=-\alpha_{2} .
$$

So, equation (1) reduces to

$$
T=-\mathrm{PI}+\mu A_{1}+\alpha_{1}\left(A_{2}-A_{1}^{2}\right) .
$$

$A_{1}$ is Rivlin-Ericksen material expansion of the strain rate tensor as the derivative rotates and translates with flow. The nonviscous squeezed flow of an unsteady second grade fluid between parallel plates segregated by a distance $z=$ $\pm l(1-\alpha t)^{1 / 2}$, where characteristic parameter is $\alpha$ and length $l$ at $t=0$ is considered. Furthermore, $\alpha>0$ is relative to the motion of both squeezed plates till they connect each other at $t=1 / \alpha$, for when $\alpha<0$, the plates are separated.

The fields of the flows corresponding to Cartesian coordinates [16] are

$$
\begin{aligned}
& V=u(x, y, t) i+v(x, y . t) j, \\
& \theta=\theta(x, y, t) .
\end{aligned}
$$

The momentum equations which govern for the problem become

$$
\begin{aligned}
& \rho\left(\frac{\partial u}{\partial t}+u \frac{\partial u}{\partial x}+v \frac{\partial u}{\partial y}\right)=-\frac{\partial P}{\partial x}+2 \mu \frac{\partial^{2} u}{\partial x^{2}}+2 \alpha_{1} \frac{\partial^{3} u}{\partial t \partial x^{2}}-\alpha_{1} \frac{\partial}{\partial x}\left(\frac{\partial v}{\partial x}\right)^{2}+\mu \frac{\partial^{2} u}{\partial y^{2}}+\mu \frac{\partial^{2} v}{\partial y \partial x}+\alpha_{1} \frac{\partial^{3} u}{\partial t \partial y^{2}} \\
& +\alpha_{1} \frac{\partial^{3} v}{\partial t \partial y^{2}}-\alpha_{1} \frac{\partial^{2} u}{\partial y \partial x} \frac{\partial u}{\partial y}-\alpha_{1} \frac{\partial^{2} u}{\partial y^{2}} \frac{\partial u}{\partial x}+\alpha_{1} \frac{\partial^{2} u}{\partial y \partial x} \frac{\partial v}{\partial x}+\alpha_{1} \frac{\partial v}{\partial x} \frac{\partial^{2} v}{\partial y \partial x}+\alpha_{1} \frac{\partial^{2} u}{\partial y^{2}} \frac{\partial v}{\partial y}+\alpha_{1} \frac{\partial u}{\partial y} \frac{\partial^{2} v}{\partial y^{2}} \\
& -\alpha_{1} \frac{\partial v}{\partial x} \frac{\partial^{2} v}{\partial y^{2}}-\alpha_{1} \frac{\partial v}{\partial y} \frac{\partial^{2} v}{\partial y \partial x} \\
& \rho\left(\frac{\partial v}{\partial t}+u \frac{\partial v}{\partial x}+v \frac{\partial v}{\partial y}\right)=-\frac{\partial P}{\partial y}+2 \mu \frac{\partial^{2} v}{\partial x^{2}}+2 \alpha_{1} \frac{\partial^{3} v}{\partial t \partial y^{2}}-\alpha_{1} \frac{\partial}{\partial y}\left(\frac{\partial u}{\partial y}\right)^{2}+\mu \frac{\partial^{2} v}{\partial x^{2}}+\mu \frac{\partial^{2} u}{\partial y \partial x}+\alpha_{1} \frac{\partial^{3} u}{\partial t \partial x \partial y} \\
& +\alpha_{1} \frac{\partial^{2} u}{\partial x^{2}} \frac{\partial v}{\partial x}+\alpha_{1} \frac{\partial^{2} v}{\partial x^{2}} \frac{\partial u}{\partial x}-\alpha_{1} \frac{\partial u}{\partial y} \frac{\partial^{2} u}{\partial x^{2}}-\alpha_{1} \frac{\partial^{2} u}{\partial y \partial x} \frac{\partial u}{\partial x}-\alpha_{1} \frac{\partial^{2} u}{\partial y \partial x} \frac{\partial v}{\partial y}-\alpha_{1} \frac{\partial u}{\partial y} \frac{\partial^{2} v}{\partial y \partial x}-\alpha_{1} \frac{\partial v}{\partial x} \frac{\partial^{2} v}{\partial y \partial x}-\alpha_{1} \frac{\partial v}{\partial y} \frac{\partial^{2} v}{\partial x^{2}} .
\end{aligned}
$$

The obtained energy equation is

$$
\begin{aligned}
\rho C_{P}\left(\frac{\partial}{\partial t}+u \frac{\partial}{\partial x}+v \frac{\partial}{\partial y}\right) T= & k\left(\frac{\partial^{2}}{\partial x^{2}}+\frac{\partial^{2}}{\partial y^{2}}\right) T-p \frac{\partial u}{\partial x}+2 \mu\left(\frac{\partial u}{\partial x}\right)^{2}+2 \alpha_{1} \frac{\partial u}{\partial x} \frac{\partial^{2} u}{\partial t \partial x}-\alpha_{1} \frac{\partial u}{\partial x}\left(\frac{\partial v}{\partial x}\right)^{2}+\mu\left(\frac{\partial u}{\partial y}\right)^{2} \\
& +\mu \frac{\partial v}{\partial x} \frac{\partial u}{\partial y}+\alpha_{1} \frac{\partial u}{\partial y} \frac{\partial^{2} u}{\partial t \partial y}+\alpha_{1} \frac{\partial u}{\partial y} \frac{\partial^{2} v}{\partial t \partial y}-\alpha_{1} \frac{\partial u}{\partial x}\left(\frac{\partial u}{\partial y}\right)^{2}+\alpha_{1} \frac{\partial u}{\partial x} \frac{\partial u}{\partial y} \frac{\partial v}{\partial x}+\alpha_{1} \frac{\partial v}{\partial y}\left(\frac{\partial u}{\partial y}\right)^{2}+\mu \frac{\partial u}{\partial y} \frac{\partial v}{\partial x}+\mu\left(\frac{\partial v}{\partial x}\right)^{2} \\
& +\alpha_{1} \frac{\partial v}{\partial x} \frac{\partial^{2} u}{\partial t \partial y}+\alpha_{1} \frac{\partial v}{\partial x} \frac{\partial^{2} v}{\partial t \partial x}-\alpha_{1} \frac{\partial v}{\partial x} \frac{\partial u}{\partial y} \frac{\partial u}{\partial x}+\alpha_{1} \frac{\partial u}{\partial x}\left(\frac{\partial v}{\partial x}\right)^{2}--\alpha_{1} \frac{\partial v}{\partial y}\left(\frac{\partial v}{\partial x}\right)^{2}-p \frac{\partial v}{\partial y}+2 \mu\left(\frac{\partial v}{\partial y}\right)^{2}+2 \alpha_{1} \frac{\partial v}{\partial y} \frac{\partial^{2} v}{\partial t \partial y}-\alpha_{1} \frac{\partial v}{\partial y}\left(\frac{\partial u}{\partial y}\right)^{2},
\end{aligned}
$$


where specific heat is $C_{P}$.

By using boundary layer flow equations, (3)-(9) become

$$
\begin{aligned}
\rho\left(\frac{\partial u}{\partial t}+u \frac{\partial u}{\partial x}+v \frac{\partial u}{\partial y}\right)= & \frac{\mu}{\rho} \frac{\partial^{2} u}{\partial y^{2}}+\frac{\alpha_{1}}{\rho} \frac{\partial^{3} u}{\partial t \partial y^{2}}-\frac{\alpha_{1}}{\rho} \frac{\partial u}{\partial y} \frac{\partial^{2} u}{\partial x \partial y} \\
& +\frac{\alpha_{1}}{\rho} \frac{\partial u}{\partial x} \frac{\partial^{2} u}{\partial y^{2}}+\frac{\alpha_{1}}{\rho} \frac{\partial v}{\partial y} \frac{\partial^{2} u}{\partial y^{2}},
\end{aligned}
$$$$
\frac{\partial T}{\partial t}+u \frac{\partial T}{\partial x}=\frac{k}{\rho C_{P}}\left(\frac{\partial^{2} T}{\partial x^{2}}+\frac{\partial^{2} T}{\partial y^{2}}\right)+\frac{\mu}{\rho}\left(\frac{\partial u}{\partial y}\right)^{2}-\frac{\alpha_{1}}{\rho} \frac{\partial u}{\partial x}\left(\frac{\partial u}{\partial y}\right)^{2} .
$$

Conforming to layout of the inquisition in Figure 1, the boundary conditions' suits for the flow are

$$
\begin{aligned}
& u=0, v=v_{w}=\frac{\mathrm{d} h}{\mathrm{~d} t}, T=T_{H} \text { at } y=h(t), \\
& \frac{\partial u}{\partial y}=0, \frac{\partial T}{\partial t}, v=0 \text { at } y=0 .
\end{aligned}
$$

2.1. Nondimensional Equations. Using transformation [11],

$$
u=\frac{\alpha x}{2(1-\alpha t)} F^{\prime}(\eta), v=\frac{-\alpha l}{2(1-\alpha t)^{1 / 2}} F(\eta),
$$

where

$$
\begin{aligned}
\eta & =\frac{y}{l(1-\alpha t)^{1 / 2}}, \\
\theta(\eta) & =\frac{T}{T_{H}} .
\end{aligned}
$$

Applying equations (15)-(17) in equations (10)-(12), we obtain

$$
\begin{gathered}
2 \lambda \mathrm{SF}^{\mathrm{IV}}+\delta^{2} F^{\prime \prime \prime}+4 \lambda^{2} F^{\prime \prime \prime}-\mathrm{SBF}^{\prime \prime} \\
-S \delta^{4}\left(\eta F^{\prime \prime}-2{F^{\prime 2}}^{2}+F F^{\prime \prime}-2 F^{\prime}\right)=0, \\
\delta^{2} \lambda^{5} \theta^{\prime \prime}-2 \eta \lambda S^{2} \operatorname{Pr} \delta \theta^{\prime}+\delta \operatorname{EcPr}\left(\lambda^{5} F^{\prime \prime 2}-2 \delta S^{2} F^{\prime} F^{\prime \prime}\right)=0,
\end{gathered}
$$

where

$$
\delta=\frac{l(1-\alpha t)}{\alpha}, S=\frac{\alpha l^{2}}{2 \mu}, \operatorname{Pr}=\frac{C_{P} \mu}{k}, \mathrm{Ec}=\frac{1}{C_{P} T_{H}}\left(\frac{\alpha x}{2(1-\alpha t)}\right),
$$

Here, nondimensional length is $\delta$, Ec is Eckert number, $S$ is squeezing number, and $\operatorname{Pr}$ is Prandtl number is dimensionless.

The nondimensional boundary conditions are

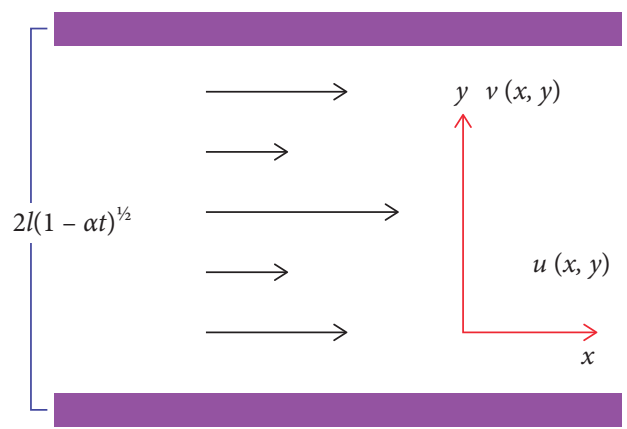

$0(u)=0(x)=0(t) 1,0(v)=0(y)=0$.

Figure 1: Physical geometry of the problem.

$$
\begin{aligned}
& F(0)=0, F^{\prime \prime}(0)=0, \\
& F(1)=1, F^{\prime}(1)=0, \\
& \theta^{\prime}(0)=0, \\
& \theta(1)=1 .
\end{aligned}
$$

\section{Numerical Solution}

The highly nonlinear partial differential equations are changed to ordinary differential equations by using transformations by shooting technique along with Runge-Kutta scheme are numerically solved with the aid of Maple software equations (34)-(45).

New variables are used to lessen the higher order ODEs into 1 st order equations, i.e.,

$$
\begin{aligned}
& F=w_{1}, F^{\prime}=w_{2}, F^{\prime \prime}=w_{3}, F^{\prime \prime \prime}=w_{4}, F^{\mathrm{IV}}=w_{4}^{\prime}, \\
& \theta=w_{5}, \theta^{\prime}=w_{6}, \theta^{\prime \prime}=w_{6}^{\prime} .
\end{aligned}
$$

New system of ODEs by using equation (23) is formed, i.e.,

$$
\begin{aligned}
w_{1}^{\prime}= & w_{2}, w_{2}^{\prime}=w_{3}, w_{3}^{\prime}=w_{4}, w_{4}^{\prime}=w_{5}, \\
w_{4}^{\prime}= & \frac{1}{2 \lambda S}\left[-\delta^{2} F^{\prime \prime \prime}-4 \lambda^{2} F^{\prime \prime \prime}+S B F^{\prime \prime}\right. \\
& \left.+S \delta^{4}\left(\eta F^{\prime \prime}-2 F^{\prime 2}+F F^{\prime \prime}-2 F^{\prime}\right)\right],
\end{aligned}
$$

$$
w_{5}^{\prime}=\frac{1}{\delta^{2} \lambda^{5}}\left[2 \eta \lambda S^{2} \operatorname{Pr} \delta \theta^{\prime}-\delta E c \operatorname{Pr}\left(\lambda^{5} F^{\prime \prime 2}-2 \delta S^{2} F^{\prime} F^{\prime \prime}\right)\right],
$$

along with boundary conditions

$$
\begin{aligned}
a_{1}(0) & =0, a_{1}(\infty)=1, a_{2}(0)=1, a_{2}(\infty) \\
& =0, a_{4}(0)=1, a_{4}(\infty)=0 .
\end{aligned}
$$




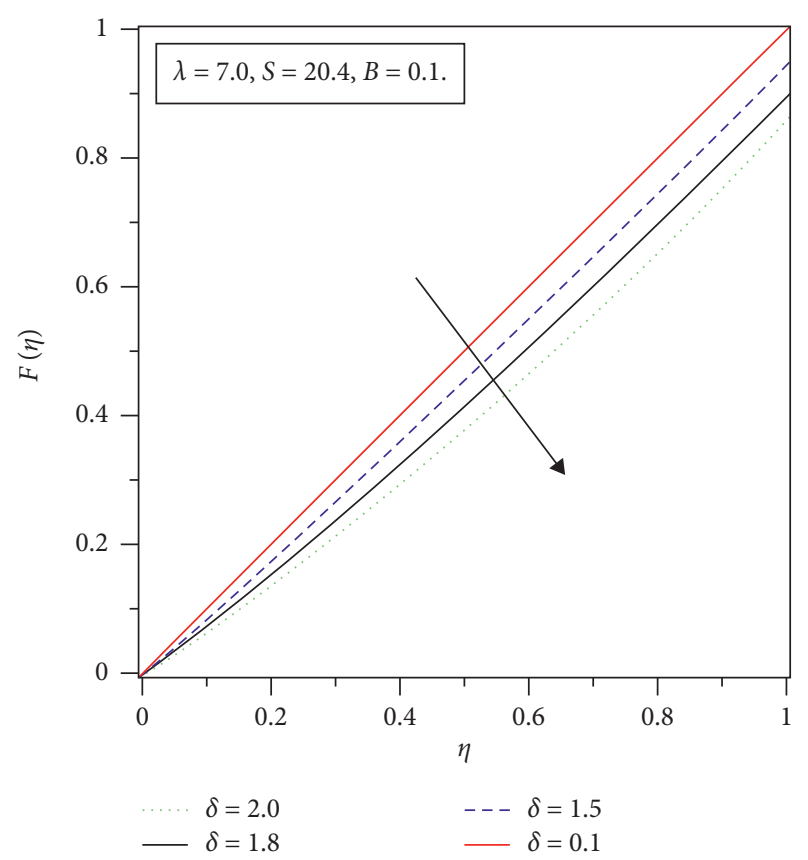

Figure 2: Impact of decreasing effects of $\delta$ for velocity portray.

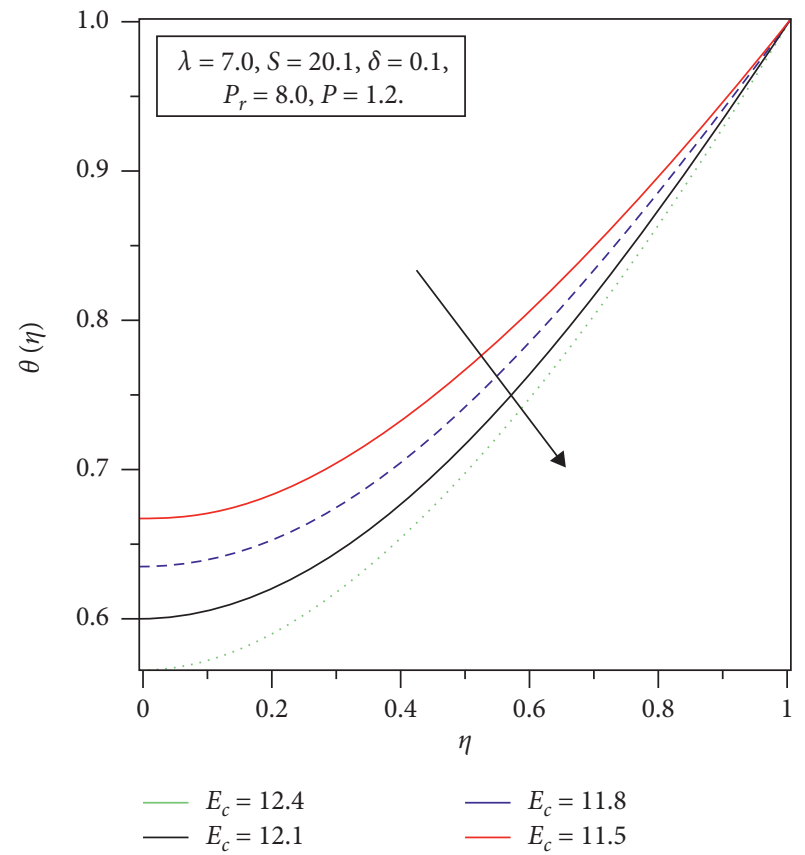

Figure 3: Illustration of decreasing effect of Ec on the temperature distribution.

\section{Graphical Discussion}

The graphical discussion of the problem described is as given below. Figure 2 shows the results of $\delta$ for velocity by assuming the values of $\delta$ as $\delta=0.1,1.5,1.8,2.0$ which give a decline in the graph. Figures 3-5 give the temperature profile for the Eckert number, Prandtl number, and squeezing

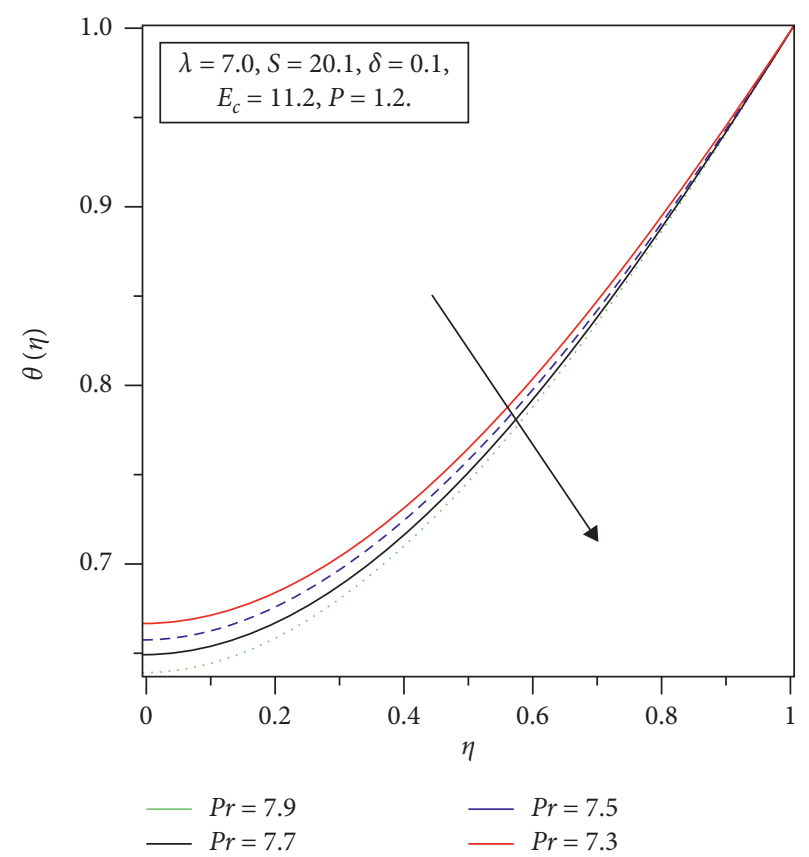

FIgURe 4: Decreasing effect of Pr on temperature portray.

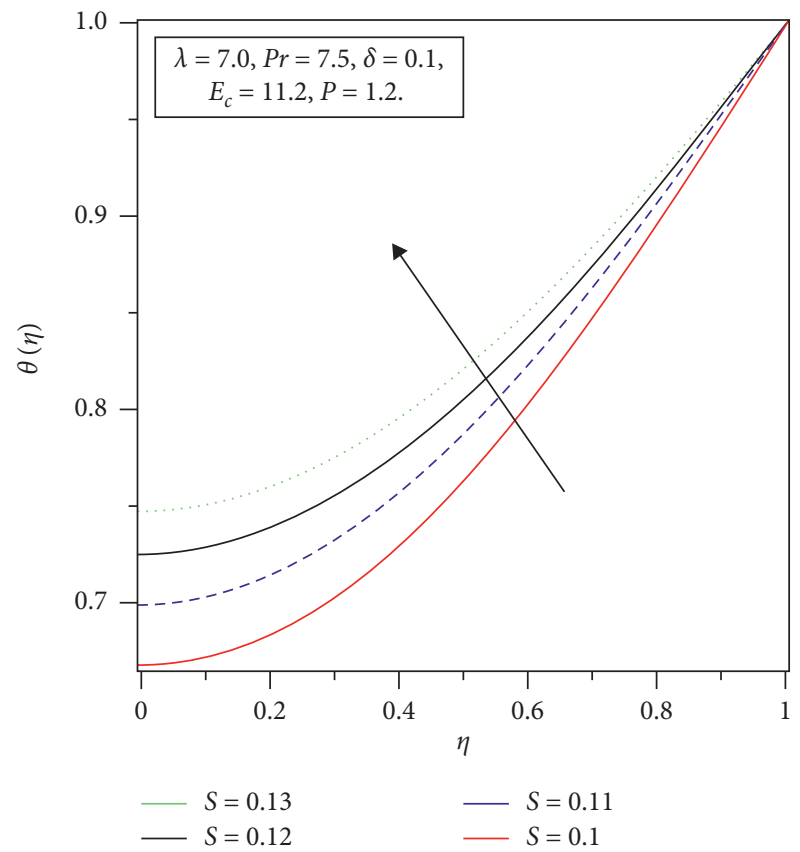

Figure 5: Influence of increasing effects of the squeezing number on temperature.

number by using different values of all numbers as Ec $=$ 11.5, 11.8, 12.1, 12.4 and $\operatorname{Pr}=7.3,7.5,7.7,7.9$, and we have used such values of the Prandtl number due to non-Newtonian fluid flow and similarly for $S=0.1,0.11,0.12,0.13$. The graph decreases for Ec and Pr while increases for the squeezing number. Figure 6 shows skin friction graph for $\lambda$ and $\delta$ for the values $\lambda=0.1,0.3,0.5$ with positively 


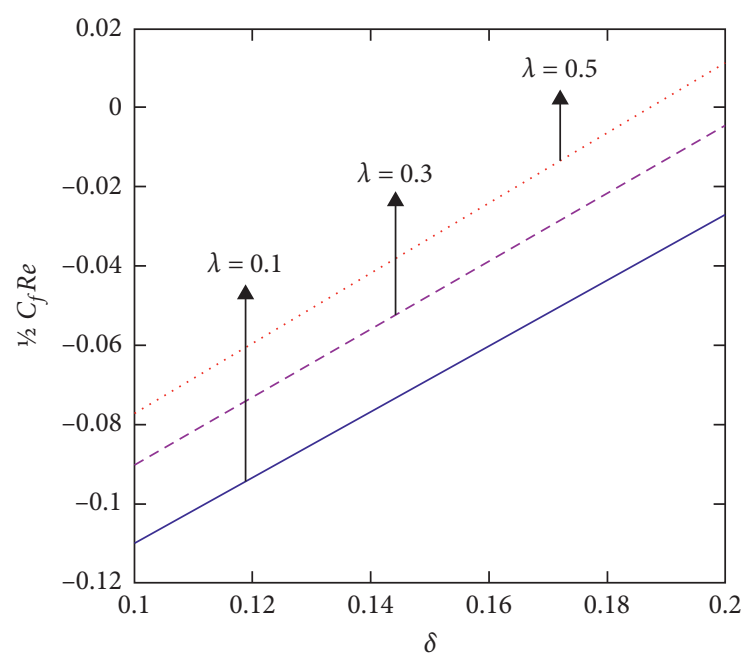

Figure 6: Increasing effects of $\lambda$ on the skin friction factor.

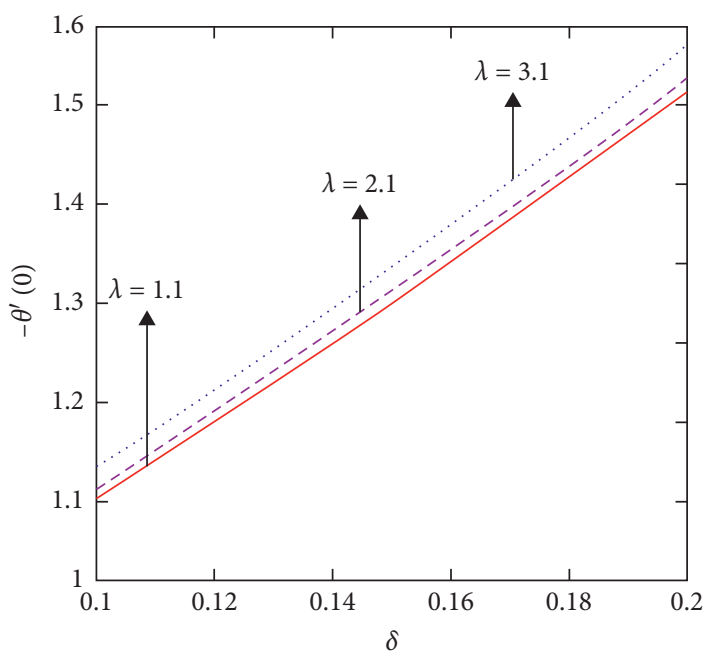

FIGURE 7: Impact of increasing effects of $\lambda$ for the Nusselt number.

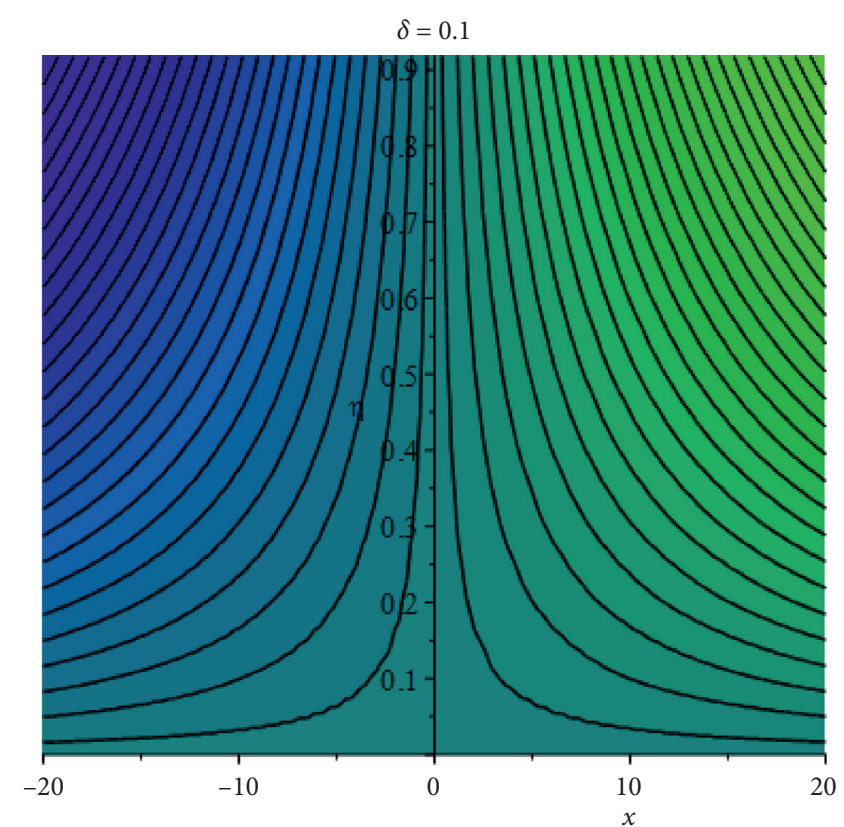

Figure 8: Stream lines for $\delta=0.1$. 


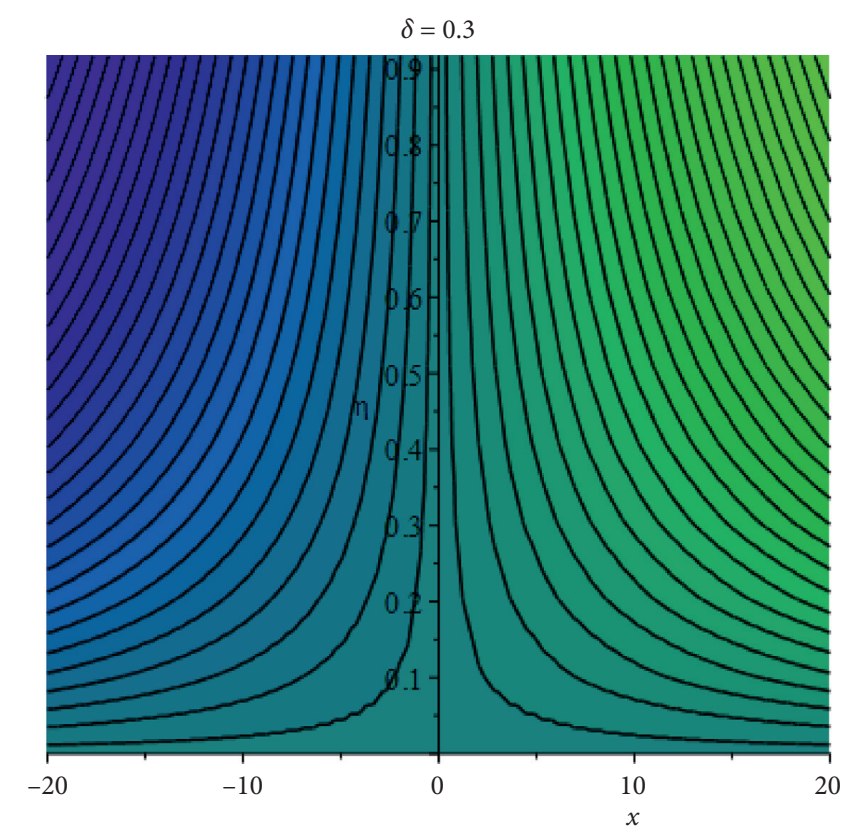

Figure 9: Stream lines for $\delta=0.3$.

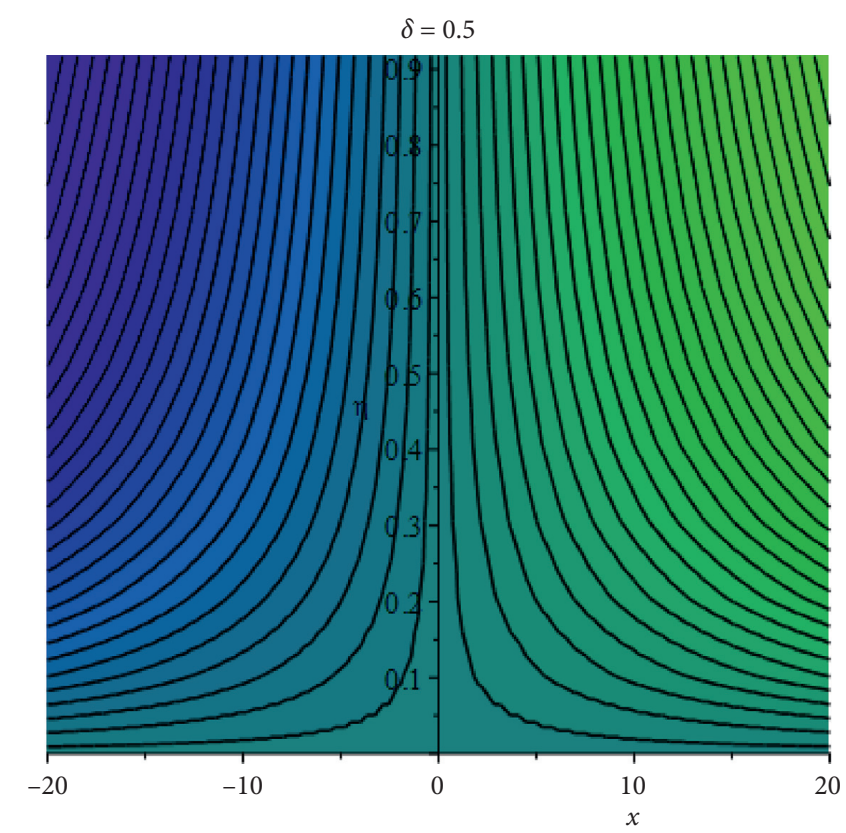

Figure 10: Stream lines for $\delta=0.5$.

increasing values. Figure 7 explicates the graphical representations of the Nusselt number for $\lambda$ and $\delta$ with $\lambda=1.1,2.1,3.1$ with increment in the graph, as the Nusselt number is the ratio of convective to conductive heat transfer at the boundary in a fluid. Figures $8-10$ illustrate the stream lines for the values of $\delta=0.1,0.3,0.5$. Figures $11-13$ demonstrate the three-dimensional graph for $\delta$. Table 1 gives different values for skin friction for $\delta, \lambda, S$, and $B$. 


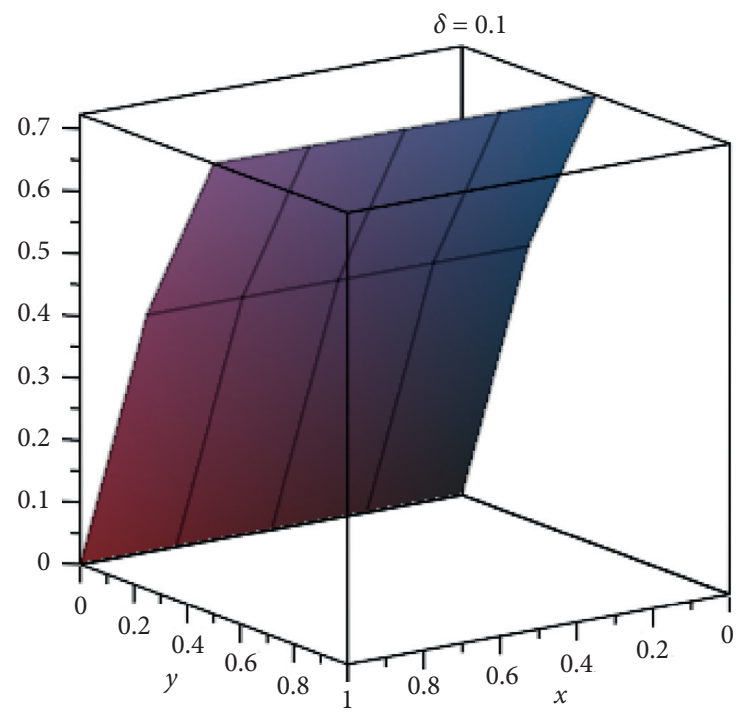

Figure 11: $3 D$ graph for $\delta=0.1$.

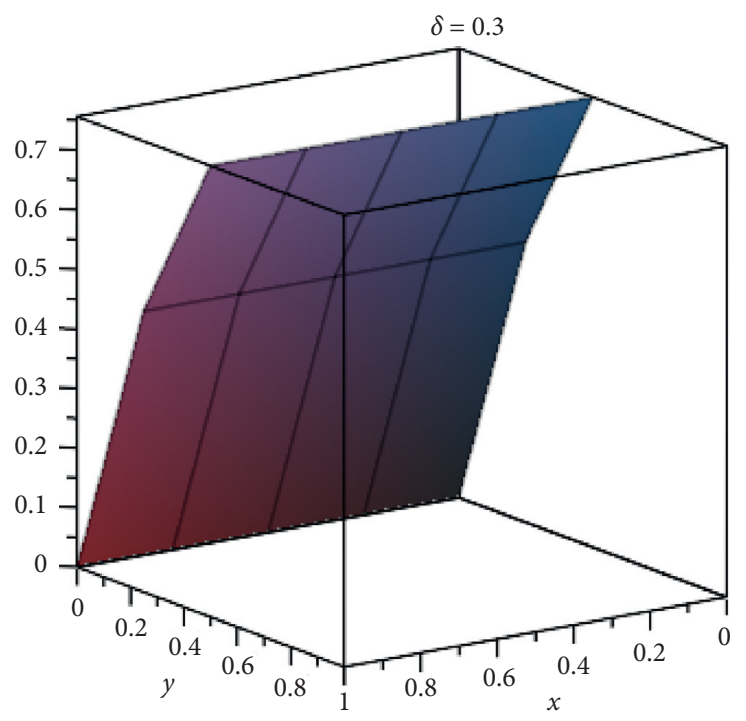

Figure 12: $3 D$ graph for $\delta=0.3$.

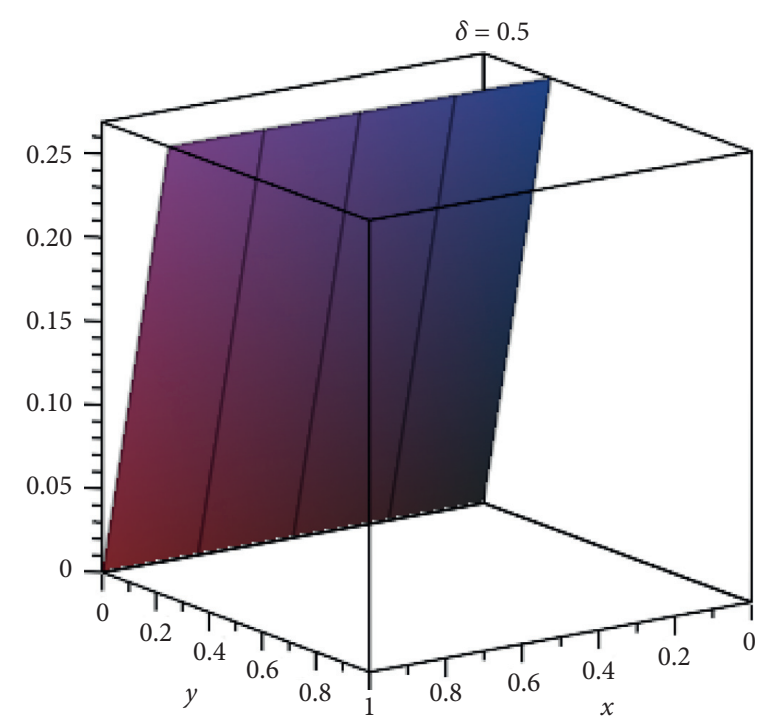

Figure 13: $3 D$ graph for $\delta=0.5$. 
TABLe 1: Values of $\delta, \lambda, S$, and $B$ for the skin friction coefficient.

\begin{tabular}{|c|c|c|c|c|c|}
\hline$\delta$ & $S$ & $\lambda$ & $B$ & $1 / 2 C_{f} \operatorname{Re}$ & [13] \\
\hline 0.1 & 20.4 & 7.0 & 0.5 & 0.99999 & -2.048052 \\
\hline 1.5 & & & & 0.84691 & -2.227279 \\
\hline 1.8 & & & & 0.71694 & -2.423537 \\
\hline \multirow[t]{10}{*}{2.0} & & & & 0.585360 & -2.048052 \\
\hline & 20.8 & & & 0.99966 & -0.938249 \\
\hline & 21.2 & & & 0.99669 & -0.630876 \\
\hline & 21.6 & & & 0.99696 & -2.048052 \\
\hline & & 7.1 & & 0.78506 & -2.238506 \\
\hline & & 7.2 & & 0.51946 & -2.451946 \\
\hline & & 7.3 & & 0.480520 & -2.048052 \\
\hline & & & 0.6 & 0.852435 & -2.056336 \\
\hline & & & 0.7 & 0.75236 & -2.057456 \\
\hline & & & 0.8 & 0.625413 & -2.048052 \\
\hline
\end{tabular}

\section{Concluding Remarks}

Non-Newtonian fluid flow is very persuasive topic since many years because it has an extensive use in many applications such as mining industry, chemical engineering, petroleum engineering, and plastic processing industry. Now, the development in industries has motivated the researchers to discover non-Newtonian fluids' properties in a more organized way. We have discussed the unsteady boundary layer flow of a second-grade fluid. The discussion is significantly influenced by the fluid which is constructed to check the obtained results which are given below:

(1) The velocity with respect to delta and temperature for Ec and Pr are decreasing

(2) We have increasing graphs of the skin friction, Nusselt number, and squeezing number of the fluid by increasing the values of their parameters

\section{Abbreviations}

$C_{p}: \quad$ Specific heat at constant pressure $J / K^{-1} \mathrm{~kg}^{-1}$

$t: \quad$ Time $[T]$

$\alpha: \quad$ Squeezed strength $[l]$

$f: \quad$ Body force $\left[N / \mathrm{m}^{3}\right]$

$P: \quad$ Pressure field $\left[\mathrm{ML} / \mathrm{T}^{2}\right]$

$\lambda: \quad$ Viscosity ratio

$T: \quad$ Temperature $[K]$

$\rho: \quad$ Density $\left[\mathrm{kg} / \mathrm{m}^{3}\right]$

$\theta: \quad$ Temperature $[K]$

Pr: Prandtl number $\mathrm{Ns} / \mathrm{m}^{2}$

$\tau$ : $\quad$ Stress tensor $N / \mathrm{m}^{2}$

$V: \quad$ Velocity of the fluid $\left[\mathrm{m}^{3} / \mathrm{s}\right]$

$\mu_{\beta}: \quad$ Viscosity of the fluid $N / \mathrm{m}^{2} \mathrm{~s}^{-1}$

$\gamma: \quad$ Dimensionless number

$N(\tau)$ : Distribution function

$A_{1}$ : Rivlin-Ericksen tensor

Ec: $\quad$ Eckert number $\mathrm{m}^{2} / \mathrm{s}$

$u, v: \quad$ Velocity components along $x, y$ direction $[L / T]$

I: Identity tensor

$x, y, z$ : Spatial coordinates $[L]$.

\section{Data Availability}

The data used to support the findings of the study are available from the corresponding author upon request.

\section{Conflicts of Interest}

The authors declare that they have no conflicts of interest.

\section{References}

[1] M. M. Rashidi, A. M. Siddiqui, and M. Asadi, "Application of homotopy analysis method to the unsteady squeezing flow of a second-grade fluid between circular plates," Mathematical Problems in Engineering, vol. 2010, Article ID 706840, 18 pages, 2010.

[2] A. Hussain, S. Akbar, L. Sarwar, and S. Nadeem, "Probe of radiant flow on temperature-dependent viscosity models of differential type MHD fluid," Mathematical Problems in Engineering, vol. 2020, Article ID 2927013, 16 pages, 2020.

[3] M. Sheikholeslami, D. Domiri Ganji, M. Younus Javed, and R. Ellahi, "Effect of thermal radiation on magnetohydrodynamics nanofluid flow and heat transfer by means of two phase model," Journal of Magnetism and Magnetic Materials, vol. 374, pp. 36-43, 2015.

[4] A. Hussain, L. Sarwar, S. Akbar, M. Y. Malik, and S. Ghafoor, "Model for MHD viscoelastic nanofluid flow with prominence effects of radiation," Heat Transfer Asian Research, vol. 48, no. 2, 2018.

[5] R. Ellahi and A. Riaz, "Analytical solutions for MHD flow in a third-grade fluid with variable viscosity," Mathematical and Computer Modelling, vol. 52, no. 9-10, pp. 1783-1793, 2010.

[6] A. Hussain, S. Akbar, L. Sarwar, S. Nadeem, and Z. Iqbal, "Effect of time dependent viscosity and radiation effcacy on a non-Newtonian fluid," Heliyon, vol. 5, no. 2, 2019.

[7] R. Ellahi and S. Afzal, "Effects of variable viscosity in a third grade fluid with porous medium: an analytic solution," Communications in Nonlinear Science and Numerical Simulation, vol. 14, no. 5, pp. 2056-2072, 2009.

[8] A. Wakif, I. L. Animasaun, P. V. Satya Narayana, and G. Sarojamma, "Meta-analysis on thermo-migration of tiny/ nano-sized particles in the motion of various fluids," Chinese Journal of Physics, vol. 68, pp. 293-307, 2020.

[9] F. Mabood, A. Shafiq, T. Hayat, and S. Abelman, "Radiation effects on stagnation point flow with melting heat transfer and second order slip," Results in Physics, vol. 7, pp. 31-42, 2017. 
[10] A. Hussain, L. Sarwar, S. Akbar, and S. Nadeem, "Mathematical model for blood flow through the stenosed channel," Physica Scripta, vol. 95, no. 2, 2019.

[11] S. Saleem, M. Qasim, A. A. Alderremy, and S. Noreen, "Heat transfer enhancement using different shapes of $\mathrm{Cu}$ nanoparticles in the flow of water based nanofluid," Physica Scripta, vol. 95, no. 5, 2020.

[12] A. Hussain, S. Akbar, L. Sarwar, and M. Y. Malik, "Numerical investigation of squeezing flow of Walters' B fluid through parallel plates," Journal of the Brazilian Society of Mechanical Sciences and Engineering, vol. 41, no. 11, 2019.

[13] O. Reynolds, "On the theory of lubrication and its application to Mr. Beauchamp tower's experiments including an experimental determination of the viscosity of olive oil," Philosophical Transactions of the Royal Society A, vol. 177, pp. 157-234, 1886.

[14] F. R. Archibald, "Load capacity and time relations for squeeze films," Journal of Lubrication Technology, vol. 78, pp. A231A245, 1956.

[15] S. Saleem and N. Sandeep, "MHD flow and heat transfer of a dusty nano fluid over a stretching surface in porous medium," Jordan Journal of Civil Engineering, vol. 4, no. 11, pp. 23-35, 2015.

[16] S. Saleem, S. Nadeem, M. M. Rashidi, and C. S. K. Raju, “An optimal analysis of radiated nanomaterial flow with viscous dissipation and heat source," Microsystem Technologies, vol. 25, no. 2, pp. 683-689, 2019.

[17] A. Naseem, A. Shafiq, L. Zhao, and M. U. Farooq, "Analytical investigation of third grade nanofluidic flow over a riga plate using Cattaneo-Christov model," Results in Physics, vol. 9, pp. 961-969, 2018.

[18] A. Shafiq, S. Jabeen, T. Hayat, and A. Alsaedi, "CattaneoChristov heat flux model for squeezed flow of third grade fluid," Surface Review and Letters, vol. 24, no. 7, Article ID 1750098, 2017.

[19] A. Shafiq, Z. Hammouch, and T. N. Sindhu, "Bioconvective MHD flow of tangent hyperbolic nanofluid with Newtonian heating," International Journal of Mechanical Sciences, vol. 133, pp. 759-766, 2017.

[20] G. Rasool, T. Zhang, A. J. Chamkha, A. Shafiq, I. Tlili, and G. Shahzadi, "Entropy generation and consequences of binary chemical reaction on MHD Darcy-Forchheimer Williamson nanofluid flow over non-linearly stretching surface," Entropy, vol. 22, no. 1, p. 18, 2020.

[21] A. Shafiq, I. Zari, G. Rasool, I. Tlili, and T. S. Khan, "On the MHD Casson axisymmetric Marangoni forced convective flow of nanofluids," Mathematics, vol. 7, no. 11, p. 1087, 2019.

[22] A. Shafiq, I. Khan, G. Rasool, A. Seikh, and E.-S. Sherif, "Significance of double stratification in stagnation point flow of third-grade fluid towards a radiative stretching cylinder," Mathematics, vol. 7, no. 11, p. 1103, 2019.

[23] G. Rasool, A. Shafiq, C. M. Khalique, and T. Zhang, "Magnetohydrodynamic Darcy-Forchheimer nanofluid flow over a nonlinear stretching sheet," Physica Scripta, vol. 94, no. 10, Article ID 105221, 2019.

[24] A. Shafiq, Z. Hammouch, and A. Turab, "Impact of radiation in a stagnation point flow of Walters' B fluid towards a Riga plate," Thermal Science and Engineering Progress, vol. 6, pp. 27-33, 2018.

[25] A. Shafiq and T. N. Sindhu, "Statistical study of hydromagnetic boundary layer flow of Williamson fluid regarding a radiative surface," Results in Physics, vol. 7, pp. 3059-3067, 2017. 